\title{
Clinical characteristics and prognostic factors of pulmonary MALT lymphoma
}

\author{
R. Borie, M. Wislez, G. Thabut, M. Antoine, A. Rabbat, L-J. Couderc, \\ I. Monnet, H. Nunes, F-X. Blanc, H. Mal, A. Bergeron, D. Dusser, \\ D. Israël-Biet, B. Crestani and J. Cadranel
}

ABSTRACT: Mucosa-associated lymphoid tissue-derived (MALT) lymphoma, a low grade B-cell extranodal lymphoma, is the most frequent subset of primary pulmonary lymphoma. Our objective was to evaluate the initial extent of disease and to analyse the characteristics and long-term outcome of these patients.

All chest and pathological departments of teaching hospitals in Paris were contacted in order to identify patients with a histological diagnosis of primary pulmonary lymphoma of the MALT subtype.

63 cases were identified. The median age was 60 yrs. $36 \%$ of cases had no symptoms at diagnosis. $46 \%$ of patients had at least one extrapulmonary location of lymphoma. The estimated 5- and 10 -yr overall survival rates were $90 \%$ and $72 \%$, respectively. Only two of the nine observed deaths were related to Iymphoma. Age and performance status were the only two adverse prognostic factors for survival. Extrapulmonary location of lymphoma was not a prognostic factor for overall survival or for progression-free survival. Treatment with cyclophosphamide or anthracyclin was associated with shorter progression-free survival, when compared with chlorambucil.

The survival data confirm the indolent nature of pulmonary MALT lymphoma. Better progression-free survival was observed with chlorambucil when compared with cyclophosphamide or anthracyclin.

KEYWORDS: Chorambucil, mucosa-associated lymphoid tissue-derived lymphoma, prognostic, pulmonary lymphoma

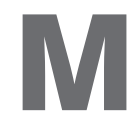

ucosa-associated lymphoid tissuederived (MALT) lymphoma is the most frequent subset of primary pulmonary lymphoma. Gastrointestinal tract involvement is the most frequent primary location, with lung location representing $15 \%$ of cases [1]. Gastric MALT lymphoma is a disseminated disease in $<25 \%$ of cases [2]. The frequency of disseminated disease in pulmonary MALT lymphoma seems to be higher [1, 3], even when it has not been specifically evaluated.

MALT lymphoma is a low grade B-cell extranodal lymphoma characterised by a proliferation of clonal marginal zone lymphocytes (MZLs) [4, 5] that invade epithelial structures and form characteristic lymphoepithelial lesions. Much evidence demonstrates that MZLs are associated with chronic antigenic stimulation, either by autoantigen or pathogen, leading to the accumulation of lymphoid tissue in involved organs [6, 7] Helicobacter pylori is the best characterised causative pathogen and is responsible for gastric MALT lymphoma [8]. Other chronic infections seem associated with MALT-derived lymphoma at other sites, even if their roles in pathogenesis are not as firmly established as that of $H$. pylori [7]. However, an association between a specific pathogen and lung MALT lymphoma has never been shown.

Patients with MALT lymphoma have a favourable Survival does not differ between gastrointestinal and non-gastrointestinal lymphoma and between localised and disseminated disease $[1,3,9]$. $H$. pylori eradication leads to the regression of gastric lymphoma in $35-75 \%$ of cases [10-12], although the standard of care for other MALT lymphomas has not been defined.

Therefore, we undertook this retrospective study in order to evaluate the initial dissemination of pulmonary MALT lymphoma and the long-term outcomes for these patients according to their clinical presentation and treatment. We also evaluated whether our patients shared some outcome with a 5-yr overall survival of $>85 \%$.

\section{AFFILIATIONS}

For affiliation details, please refer to the Acknowledgements section.

\section{CORRESPONDENCE}

J. Cadranel

Service de Pneumologie et

Réanimation

Hôpital Tenon

4 rue de la Chine 75970 cedex 20 Paris

France

E-mail: jacques.cadranel@

tnn.aphp.fr

Received:

March 092009

Accepted after revision:

June 102009

First published online:

June 182009 
characteristics leading to a common pathogenesis in pulmonary MALT lymphoma.

\section{PATIENTS AND METHODS}

All chest departments in adult teaching hospitals in Paris were contacted $(n=15)$ in order to identify patients who had been diagnosed as having pulmonary MALT lymphoma between January 1993 and February 2008. Moreover, the pathology departments attached to each hospital were asked to report their cases of histological pulmonary MALT lymphoma diagnosed during the same period of time.

\section{Inclusion criteria}

To be included in the study, patients had to have an initial pulmonary lesion assessed by computed tomography (CT) scan without extrapulmonary node involvement or chronic lymphocytic leukaemia, the diagnosis of MALT being histologically proven, according to the World Health Organization classification [4, 13], from a pathological lung lymphoma specimen. Exclusion criteria were as follows: 1) presentation of another histological type of lymphoma, including diffuse large B-cell lymphoma; 2) pulmonary relapse of MALT lymphoma without any evidence of an initial pulmonary lesion.

The clinical charts of the patients were reviewed by one of us (R. Borie) to check all the inclusion criteria. Data were collected on a standardised and anonymous collection form. For this retrospective, observational, noninterventional analysis of medical records, French law does not require specific approval of an internal review board or the consent of patients.

\section{Data collection}

The following data were collected at the time of diagnosis: clinical parameters (age, sex, other diseases, presence of " $\mathrm{B}$ " symptoms, delay between first symptoms and diagnosis, complete physical examination), performance status evaluated according to the Eastern Cooperative Oncology Group (ECOG), and biological parameters (serum lactate dehydrogenase (LDH), serum protein electrophoresis and blood cell count).

Staging was evaluated by CT scan of the thorax, abdomen and pelvis, endoscopic examination of the gastrointestinal tract with systematic biopsies, including evaluation of $H$. pylori infection status, and bone marrow biopsy. All chest CT scans were analysed by radiologists attending the various chest departments from the teaching hospitals and were reviewed by one of us (R. Borie). Pulmonary abnormalities were interpreted as previously described [14]. Pathological reports were centrally reviewed by a pathologist expert in oncology (M. Antoine).

Outcome data were collected at 3, 6 and 12 months and every 6 months thereafter until death or the last scheduled visit. Responses to treatment were based on the original data recorded by the local physician. Evaluation after treatment was classified as recommended by the 2007 International Working Group criteria for malignant lymphoma [15]. Complete remission was defined as the disappearance of all clinical, biological and morphological evidence of lymphoma. Partial remission was defined as a decrease in tumour volume $>50 \%$, as assessed by CT scan. Disease control was defined as the sum of partial and complete remission. Progression was defined as the appearance of any new lesion or $a \geqslant 50 \%$ increase in tumour volume [15]. The transformation of MALT lymphoma to diffuse large B-cell lymphoma had to be demonstrated histologically, and referred to the presence of $50 \%$ blast cells or the presence of sheets of large cells associated with high mitotic activity [1]. For statistical analysis, treatments were regrouped as follows: 1) chlorambucil group, i.e. patients treated by chlorambucil-based chemotherapy (chlorambucil alone or combined with rituximab); 2) cyclophosphamide group, i.e. patients treated by cyclophosphamide-based chemotherapy (cyclophosphamide alone or combined with vincristine and/or rituximab); 3) anthracycline/fludarabine group, i.e. patients treated by anthracycline- or fludarabine-based chemotherapy with or without rituximab; 4) local therapy group, i.e. patients treated by surgery or radiotherapy; and 5) abstention.

\section{Statistical analysis}

Continuous variables were expressed as median (range) and compared by the Mann-Whitney U-test. Categorical variables were expressed as counts and proportions and compared by the Fisher's exact test. Progression-free survival (PFS) was defined from diagnosis until lymphoma progression, relapse or death. Overall survival was defined as the time from diagnosis (first biopsy) to death or the last follow-up [15]. Survival was assessed using the Kaplan-Meier estimator. We used the Cox proportional hazard model to assess the impact of variables on survival, in univariate and multivariate analysis. For the multivariate model, we considered all variables significant in the univariate analysis at the $20 \%$ level, as well as all variables known to be clinically relevant (age and performance status). All tests were two-sided, with $\mathrm{p}<0.05$ indicating statistical significance. All the analyses involved use of the software R (www.r-project.org; Bell Laboratories, Murray Hill, NJ, USA).

\section{RESULTS}

All 15 centres participated in the study. Four of the 15 centres did not have any eligible cases. 96 cases of pulmonary lymphoma were reported between January 1, 1993 and February 1,2008, of which 33 were excluded: 17 because of incomplete initial data (histology or initial thorax CT scan missing), three because of lack of lung involvement evidenced at initial diagnosis and 13 because of inadequate histology. 63 patients completed the inclusion criteria and were therefore evaluated.

\section{Clinical characteristics of the population at initial presentation}

Table 1 shows the main clinical characteristics of the patients at presentation. 10 patients had autoimmune disease at diagnosis, including systemic lupus erythematosus $(n=2)$, rheumatoid arthritis $(n=1)$ and Sjogren's syndrome $(n=5)$, and four more patients had undifferentiated connective tissue disease similar to Sjogren's syndrome [16]. None of the evaluated patients was infected by HIV ( 0 out of 42 tested), hepatitis B virus (HBV) (0 out of 38 tested) or hepatitis C virus (HCV) (0 out of 38 tested).

23 patients $(36 \%)$ had radiological abnormalities without respiratory or B symptoms. Dry cough and dyspnoea were the most frequent respiratory symptoms, found in $26(41 \%)$ and $22(35 \%)$ patients, respectively. Nine patients had prior history of pulmonary infection, including tuberculosis in four 


\begin{tabular}{lc}
\hline TABLE 1 & $\begin{array}{l}\text { Main clinical and biological characteristics of the } \\
63 \text { patients }\end{array}$ \\
\hline Characteristics & Value \\
\hline Age yrs & $60(24-83)$ \\
Females & $29(47)$ \\
Active or former tobacco use & $24(37)$ \\
Respiratory tract infection & $6(9)$ \\
$\quad$ Including tuberculosis & $4(6)$ \\
Autoimmune background & $10(16)$ \\
Respiratory symptoms & $37(58)$ \\
B symptoms & $14(22)$ \\
Cytopenia & $12(19)$ \\
LDH level more than twice the upper limit & $2(3)$ \\
\hline
\end{tabular}

Data are presented as or median (range) or $\mathrm{n}(\%)$. B symptoms include weight loss, fever and night sweats. LDH: lactate dehydrogenase.

of them. Weight loss was observed in eight (12\%) patients, fever in six $(9 \%)$ and night sweats in three $(5 \%)$. The median (range) duration between the first symptoms (or radiological abnormalities) and diagnosis was 9.0 (0.4-229.2) months. However, the median duration for diagnosis was significantly longer in patients without symptoms (27.4 (0.4-229.2) months) than those with symptoms (4.7 (0.5-62.2) months; $\mathrm{p}=0.001)$.

Blood cell counts and LDH serum level were available for all patients. 16 patients had cytopenia at diagnosis, including 10 with anaemia (range $8.6-11.9 \mathrm{~g} \cdot \mathrm{dL}^{-1}$ ), four with thrombocytopenia (range 131,000-149,000 $\mathrm{mL}^{-1}$ ), and two with mixed thrombocytopenia and anaemia. Two patients had elevated LDH serum levels more than twice the upper limit. Serum protein electrophoresis was available in 62 cases, of which 20 $(32 \%)$ showed a monoclonal immunoglobulin (Ig), with a median weight of the monoclonal peak of $4.5(1-20) \mathrm{g} \cdot \mathrm{L}^{-1}$. Monoclonal immunoglobulin was characterised in 19 cases, among which there were $16 \kappa$ and three $\lambda$ light chains and $12 \mu$, six $\lambda$ and one $\alpha$ heavy chains.

\section{Pathological diagnosis of pulmonary MALT Iymphoma}

Pathological diagnosis of pulmonary MALT lymphoma was obtained by bronchial biopsies in 19 cases (19 out of 61 performed), transbronchial biopsies in 23 cases (23 out of 26 performed), CT-guided percutaneous lung biopsies in eight cases (eight out of 10 performed), and surgical biopsy in 18 cases (18 out of 18 performed). Surgical supplementary specimens were obtained in nine more cases because of curative surgery (fig. 1). Finally, a pathological specimen from an extrathoracic MALT lesion was also obtained in 26 out of 63 patients (see below).

All pathological samples showed dense proliferation of small lymphocytes infiltrating the lung and the architecture was deeply reworked in 30 cases. Characteristic lymphoepithelial lesions containing B cells (CD20 or CD19 expression) were observed in 61 cases $(96 \%)$. B cells were confirmed in 27 cases by negative CD3 staining and lymphoepithelial lesion was better identified by cytokeratin staining in 17 cases. The neoplastic cells were morphologically like centrocytes in $75 \%$ of samples and monocytoid in $25 \%$ of samples. Plasma cells were observed in $44 \%$ of cases. Large cells were absent in 42 samples $(66 \%)$ and less than $20 \%$ in the other samples. Evaluation of monotypic expression was interpretable in 15 cases, among which there were nine $\kappa$ and five $\lambda$ light chains, six $\mu$ and two $\gamma$ heavy chains, and one and seven uninterpretable results. When performed CD5 (0 out of 37), CD10 (0 out of 27$), C D 23$ ( 0 out of 15$), \operatorname{IgD}(0$ out of 10$)$ and CD30 (0 out of six) staining were always negative. Expression of bcl2 was performed in 12 cases and positive in nine (75\%).

In this series, Epstein-Barr virus expression evaluated by in situ hybridisation for Epstein-Barr encoded RNAs was always negative $(n=5)$.

\section{Thoracic and extrathoracic evaluation}

All patients underwent thoracic CT scan, among which 33 $(52 \%)$ revealed unilateral disease involvement, limited to one lobe in $25(39 \%)$ cases. There was no topographic predominance. Mediastinal centimetric lymph nodes were observed in $10(16 \%)$ cases and pleural effusion in seven $(11 \%)$, respectively. According to CT scan analysis, 53 (84\%) patients presented multi-pattern abnormalities; consolidations $(n=35$; $55 \%)$, nodules $(n=35 ; 55 \%)$ and masses $(n=34 ; 54 \%)$ being the most frequent patterns. $54(86 \%)$ patients had airways within the lesions, and the air bronchogram seemed to be dilated in 42 $(77 \%)$ of them. Micronodules (22\%), ground-glass opacities $(27 \%)$ and septal lines (12\%) were less frequent. It is noteworthy that CT scan pattern was a solitary pulmonary nodule in only five $(8 \%)$ patients.

$29(46 \%)$ patients had at least one extrapulmonary location of lymphoma, which was unique for 22 of them (table 2). An extrapulmonary location of lymphoma was observed more frequently in patients with serum monoclonal Ig (14 out of 20) than in patients without monoclonal immunoglobulin (14 out of $42 ; \mathrm{p}=0.01) .57$ patients had systematic gastric endoscopies with histological examination, showing lymphoma involvement in 15 cases $(36 \%)$. Prevalence of histological H. pylori infection was not significantly different in cases with gastric involvement (five out of $15,33 \%$ ) than in those without (10 out of $42,24 \%$; $=0.7) .58$ patients had bone marrow biopsies, showing lymphoma involvement in eight cases (14\%).

15 patients had a ${ }^{18} \mathrm{~F}$-2-fluoro-2-deoxy-D-glucose (FDG)-positron emission tomography (PET) scan. A mild uptake was identifiable in the lung location for $12(80 \%)$ patients. In three patients, no uptake was identifiable in the place of the lymphoma lesion. The PET scan identified an ignored lymphoma location in two patients, which was confirmed by histology (nodal, $\mathrm{n}=1$; skin, $\mathrm{n}=1$ ).

\section{Treatments and outcomes}

Treatments and the results of these treatments are summarized in table 3. Six (9.5\%) patients did not receive any treatment. 20 $(32 \%)$ patients were treated only by local therapy (surgical resection, $\mathrm{n}=19$; radiotherapy, $\mathrm{n}=1)$ and $37(58.5 \%)$ only by chemotherapy. Anthracyclin-based chemotherapy was administered for almost 5 months, cyclophosphamide and chlorambucil for almost 15 months. The disease control rate was significantly better in patients from the chlorambucil group than in those from the cyclophosphasmide group $(\mathrm{p}=0.02$ 


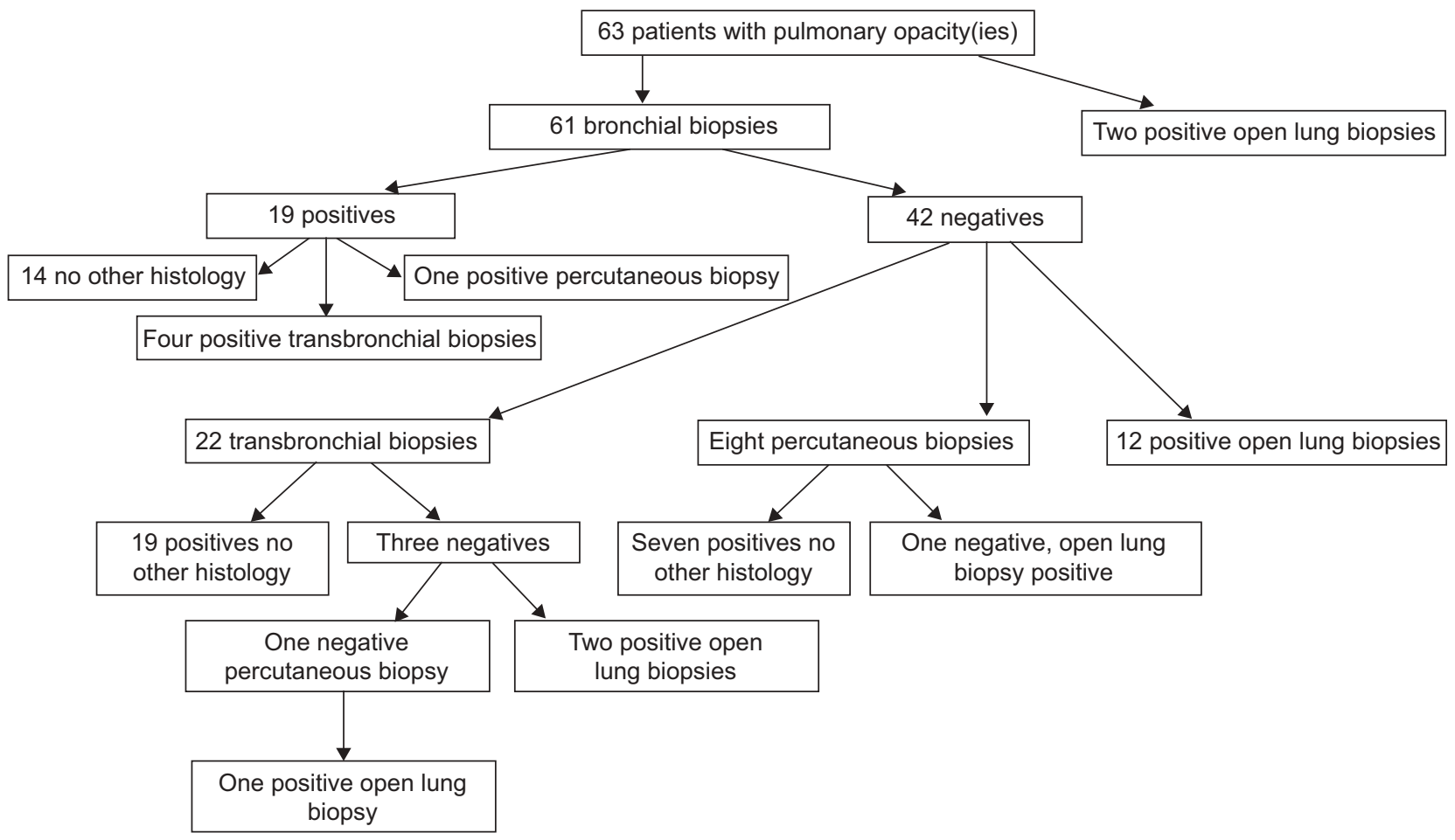

FIGURE 1. Strategy for pathological diagnosis in pulmonary mucosa-associated lymphoid tissue-derived (MALT) lymphoma. 63 patients were referred for diagnosis of pulmonary opacity(ies). 61 had bronchial biopsies during fibreoptic bronchoscopy, among which transbronchial biopsies were performed in the same procedure in 26 cases (42.6\%). In a second step, 10 patients had computed tomography (CT)-guided percutaneous transparietal biopsies. One supplementary patient had CT scan biopsies, whereas re-reading of the bronchial biopsy revealed MALT Iymphoma. In a third step, the diagnosis was made by open lung biopsy in 18 patients.

\begin{tabular}{ll} 
TABLE 2 & $\begin{array}{l}\text { Staging of the } 63 \text { patients with pulmonary } \\
\text { mucosa-associated lymphoid tissue-derived } \\
\text { (MALT) lymphoma }\end{array}$ \\
Pulmonary CT scan evaluation ${ }^{\#}$ & \\
Unilateral & $33(52)$ \\
One lobe & $25(39)$ \\
Mediastinal adenopathy & $10(16)$ \\
Pleural effusion & $7(11)$ \\
Extrapulmonary evaluation & \\
Total & $29(46)$ \\
Mucosal site & $19(30)$ \\
Stomach & $15(19)$ \\
Skin & $2(3)$ \\
Bowel & $2(3)$ \\
Conjunctiva & $1(1.5)$ \\
Cavum & $1(1.5)$ \\
Parotid & $1(1.5)$ \\
Lymphoid organs & $14(22)$ \\
Bone marrow & $8(13)$ \\
Nodal & $4(6)$ \\
Spleen & $4(6)$ \\
\hline
\end{tabular}

Data are presented as $n(\%)$. The sum of each location is higher than the total because seven patients had more than one extrapulmonary location. \#: mediastinal adenopathy or pleural effusion were assessed by computed tomography (CT) scan, and not histologically proven; " : except for spleen, all extrapulmonary locations of MALT were histologically proven. versus chlorambucil), but not significantly different from those in the anthracyclin/fludarabine group $(p=0.07$ versus chlorambucil).

With a median (range) follow-up for patients of 47 (1192) months, the estimated 5- and 10-yr PFS rates were $51 \%$ and $36 \%$, respectively (fig. 2). The proportion of patients with relapse and/or progression by treatment groups is shown in table 4 . It occurred in a known primary involved site in $16(66 \%)$ cases and in a new site in seven $(33 \%)$ cases. Factors associated with an increased probability of shorter PFS are shown in table 4. The presence of mediastinal adenopathy was associated with a higher probability of shorter PFS in the univariate analysis (hazard ratio (HR) 3.5, 95\% CI 1.3-9; $\mathrm{p}=0.01$ ), but was not confirmed in multivariate analysis $(p=0.99)$. Patients from the cyclophosphamide group (HR 6.1, 95\% CI 2-18.6; $\mathrm{p}=0.001$ ) and the anthracyclin/fludarabine group (HR 4.4, 95\% CI 1.3-15.0; $p=0.02$ ) had a higher probability of relapse than those from the chlorambucil group (HR 1). Multivariate Cox analysis confirmed that treatments by non-chlorambucil chemotherapy were independent factors associated with shorter PFS (table 4). Neither age, sex, performance status, extrapulmonary nor medullar location, nor a delay of diagnosis, were predictors of PFS.

The median PFS was not achieved in the abstention and local therapy groups with a median follow-up of 12.5 and 68.2 months, respectively. The median PFS was 8.2 yrs in the chlorambucil group, whereas it was only 0.7 yrs in both the cyclophosphamide (HR 6.1, 95\% CI 2-18.6; $\mathrm{p}=0.001$ ) and the fludarabine/anthracyclin (HR 4.4, 95\% CI 1.3-15.0; $\mathrm{p}=0.02$ ) groups (table 3). 
TABLE 3 Remission and outcome according to type of therapy

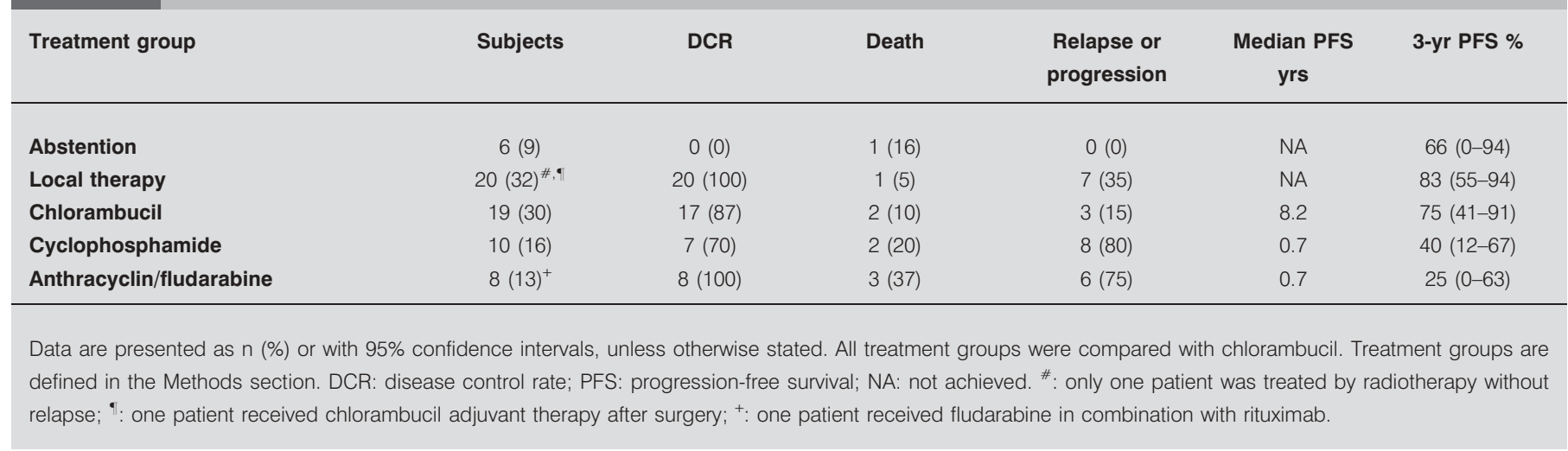

Death occurred in nine patients but was related to diffuse large B-cell lymphoma transformation of MALT in only two cases. However, it is noteworthy that four of the seven lymphomaunrelated deaths were due to secondary solid tumours (urinary bladder, kidney, gastric and colonic). The estimated 5- and 10-yr overall survival rates were $90 \%$ and $72 \%$, respectively (fig. 3). Age $>60$ yrs (HR 12.5, 95\% CI 1.5-104.0) and performance status (status 2 and 3; HR 38.6, 95\% CI 4-374) were the only two variables associated with shorter survival in the univariate analysis (table 5). Overall survival was not statistically modified by extrapulmonary location or the type of treatment (table 5). Multivariate Cox analysis (including age, performance status and extrapulmonary location) confirmed that age $>60$ yrs and performance status were independently associated with shorter overall survival $(p=0.01$ and $p=0.02$, respectively).

\section{DISCUSSION}

This study describes the largest clinical series of pathologically proven pulmonary MALT lymphoma, the most frequent type of non-Hodgkin lymphoma involving the lung. Compared

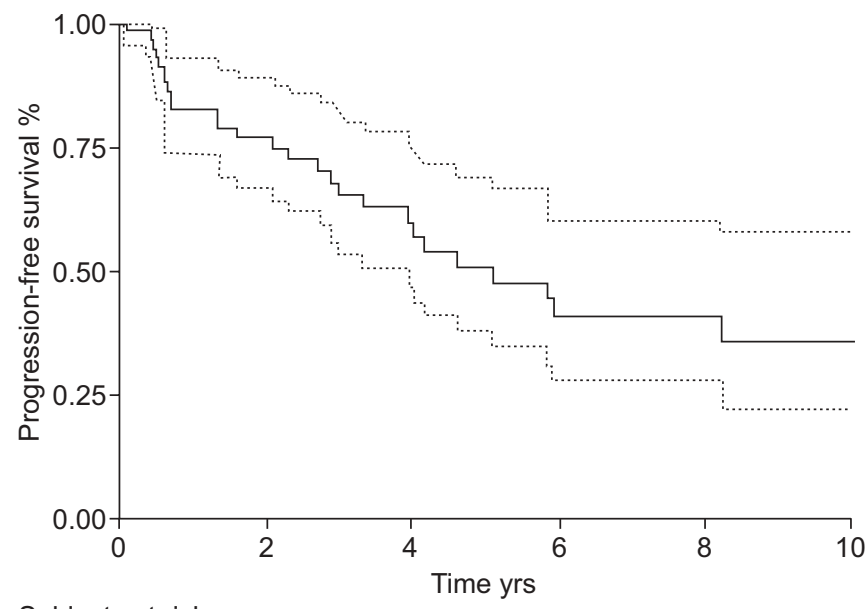

Subjects at risk $n$

63

37

22

13

9

3

FIGURE 2. Kaplan-Meier estimate of the progression-free survival (PFS) of 63 patients with pulmonary mucosa-associated lymphoid tissue-derived lymphoma. The estimated PFS was 36\% (19-52\%) at $10 \mathrm{yrs}$. ..... 95\% confidence interval. with older series $[17,18]$, most of our patients $(71.4 \%)$ were diagnosed by minimally invasive procedures, including fibreoptic bronchoscopy, bronchial and transbronchial biopsies, and CT-guided percutaneous transthoracic biopsies. This minimal diagnosis approach seems justified as most patients had multilobar $(61 \%)$ or disseminated disease $(44 \%)$ and an excellent prognosis whatever the therapeutic strategy, including abstention, surgery or single-agent chemotherapy (see below). Furthermore, it has been facilitated by the use of new biomarkers, including anti-cytokeratin antibody, to identify lymphoepithelial lesions, and CD20/CD19 and CD5 antibodies to identify marginal zone B-cells using paraffin-embedded tissue specimens [19]. Indeed, in the present series, almost all pathological reports showed lymphoepithelial lesions containing dense proliferations of B-cells. The neoplastic cells were morphologically described as centrocyte-like or monocytoidlike in almost all cases, and large cells were either absent or less than $20 \%$. Plasma cells were also found in $44 \%$ of cases.

In this study population of 63 patients, $44 \%$ of patients had a disseminated disease at diagnosis, which appears greater than the $10-34 \%$ previously observed in nongastric MALT lymphoma $[1,3,20]$. By contrast, gastric MALT lymphoma seems a confined disease in $\sim 90 \%$ of patients [2]. According to ISAACSON and WRIGHT [5], the MALT concept relies on a particular behaviour in which dissemination occurs late and after many local relapses. Indeed, the great incidence of disseminated disease in MALT lymphoma of the lung could be explained by the long delay in diagnosis, which can be $>25$ months in clinically asymptomatic patients.

There is still controversy about the utility of the PET scan in evaluating dissemination in MALT lymphoma. MALT lymphoma with indolent growth was expected to have little FDG uptake. In this series, the FDG-PET scan was positive in 12 of 15 patients with lung location, two of four with gastric location, and 0 of two with medullar location, suggesting that FDG uptake might be site specific [18]. Indeed, its sensitivity has been reported as 50-89\% [21, 22] and $83-100 \%$ [18] for stomach and lung involvement, respectively. However, the PET scan also detected unsuspected extrapulmonary lymphoma lesions in two patients (nodal and cutaneous). Altogether, these findings suggest the need for prospective evaluation of PET scans for the initial staging and medical management of pulmonary MALT lymphoma. 
TABLE 4 Hazard ratios for progression-free survival

\begin{tabular}{|c|c|c|c|c|}
\hline & Hazard ratio $(95 \% \mathrm{Cl})$ & $\mathrm{p}$-value & Hazard ratio $(95 \% \mathrm{Cl})$ & p-value \\
\hline Age $>60 \mathrm{yrs}$ & $1.0(0.5-2.2)$ & 0.9 & $0.8(0.3-1.7)$ & 0.52 \\
\hline Male sex & $0.7(0.3-1.5)$ & 0.3 & & \\
\hline \multicolumn{5}{|l|}{ ECOG } \\
\hline $2-3$ & $1.99(0.8-5)$ & 0.1 & $3.0(1.0-9.5)$ & 0.05 \\
\hline Diagnosis delay (per 1-month increase) & $1.0(0.99-1.01)$ & 0.8 & & \\
\hline Extrapulmonary location & $1.4(0.7-3.0)$ & 0.4 & $0.9(0.4-2.2)$ & 0.86 \\
\hline Gastric & $1.3(0.6-3.0)$ & 0.6 & & \\
\hline Nodal & $0.7(0.1-5.1)$ & 0.7 & & \\
\hline Medullar & $1.1(0.3-3.7)$ & 0.9 & & \\
\hline Pleural effusion & $1.7(0.6-4.9)$ & 0.7 & & \\
\hline \multicolumn{5}{|l|}{ Treatment } \\
\hline Chlorambucil & 1 & & 1 & \\
\hline Cyclophosphamide-based chemotherapy & $6.1(2-18.6)$ & 0.001 & $6.1(1.6-23.7)$ & 0.01 \\
\hline Anthracyclin or fludarabine-based chemotherapy & $4.4(1.3-15.0)$ & 0.02 & $4.9(1.3-17.6)$ & 0.02 \\
\hline Local therapy & $1.0(0.3-3.2)$ & 0.9 & $1.1(0.3-3.8)$ & 0.96 \\
\hline Abstention & $1.2(0.1-10.1)$ & 0.8 & $1.2(0.1-11)$ & 0.84 \\
\hline
\end{tabular}

All treatment groups were compared with chlorambucil. Multivariate Cox analysis was performed using significant clinical variables. Treatment groups are defined in the Methods section. ECOG: Eastern Cooperative Oncology Group.

Marginal zone lymphoma is considered to be a model of antigen-driven lymphoma, driven either by autoantigens or by microbial pathogens [7]. The risk of developing lymphoma is estimated to be 6.6-44 times higher in Sjogren's syndrome than in the normal population [23, 24], with marginal zone

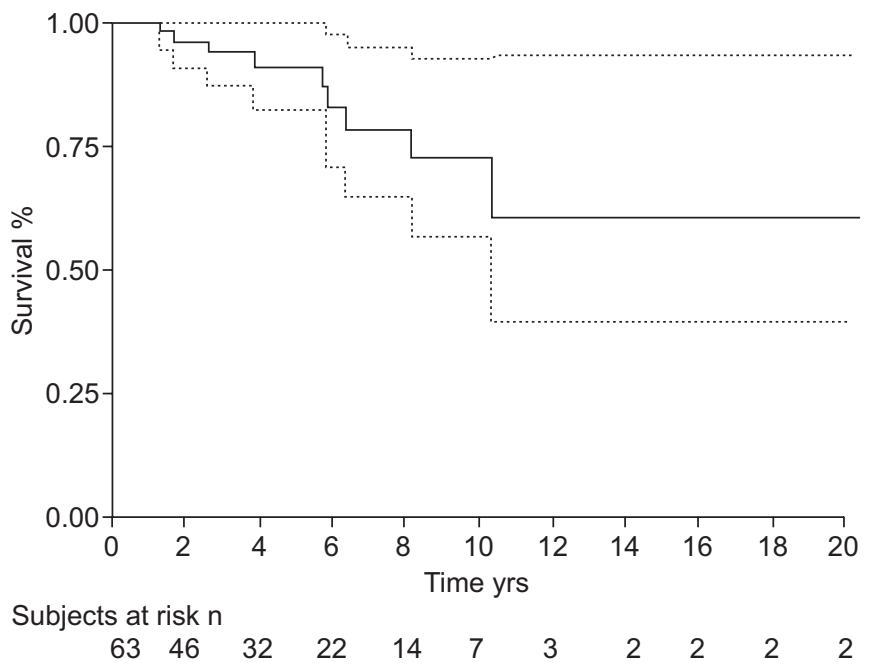

FIGURE 3. Kaplan-Meier estimate of the overall survival of 63 patients with pulmonary mucosa-associated lymphoid tissue-derived lymphoma. The estimated overall survival of patients was $72 \%(50-86 \%)$ at $10 \mathrm{yrs}$. ..... $95 \%$ confidence interval. lymphoma being the most frequent histological subtype of lymphoma [6, 24]. In this series, 10 patients had an autoimmune disease, confirming the frequent association between these two disorders [6]. Chronic infections also seem responsible for sitespecific marginal zone lymphomas, such as with $H$. pylori for stomach, Campylobacter jejuni for small intestine, Borrelia burgdorferi for skin, Chlamydiae psittaci for ocular adnexa, and $\mathrm{HCV}$ for spleen. Moreover, other infections, i.e. Mycobacterium tuberculosis [25], EBV [26] and HHV8 [27], are involved in highgrade lymphoma with primary thoracic location. However, none of our cases was associated with HIV, HBV or HCV infection, and only a few of them had a prior pulmonary infection, including tuberculosis sequel. Prevalence of H. pylori infection was not significantly different in patients with gastric involvement $(33 \%)$ compared with those without $(24 \%)$. As the prevalence of $H$. pylori infection in the general population is only $18 \%$ [28], we cannot definitively exclude that few of our patients had $H$. pylori-driven primary gastric MALT lymphoma with secondary pulmonary dissemination. In this series, histological examination revealed absence of EBV for every patient tested. This result is in accordance with previously published series of gastric MALT lymphoma showing the absence of an association with EBV in the absence of a large B-cell lymphoma component [29]. At the present time, pulmonary MALT remains orphaned from a specific driving pathogen or perhaps does not depend on chronic infection to maintain lymphomagenesis.

Although a recent series of MALT lymphoma among which only 10 cases with lung location has suggested a lower overall 


\begin{tabular}{|c|c|c|}
\hline & \multicolumn{2}{|c|}{ Univariate analysis } \\
\hline & Hazard ratio $(95 \% \mathrm{Cl})$ & p-value \\
\hline Age $>60$ yrs & $12.5(1.5-104.0)$ & 0.02 \\
\hline Male sex & $0.7(0.2-2.5)$ & 0.5 \\
\hline \multicolumn{3}{|l|}{ ECOG } \\
\hline 0 & 1 & \\
\hline 1 & $20.3(2.1-190.4)$ & 0.008 \\
\hline $2-3$ & $38.6(4-374)$ & 0.002 \\
\hline $\begin{array}{l}\text { Diagnosis delay (per } 1 \text { month } \\
\text { increase) }\end{array}$ & $1.0(0.99-1.02)$ & 0.8 \\
\hline Extrapulmonary location & $2.2(0.6-8.4)$ & 0.2 \\
\hline Gastric & $1.8(0.4-7.8)$ & 0.4 \\
\hline Nodal & $6.0(0.6-58.8)$ & 0.1 \\
\hline Medullar & & $0.52^{\#}$ \\
\hline \multicolumn{3}{|l|}{ Pulmonary extension } \\
\hline Mediastinal adenopathy & $2(0.2-17.7)$ & 0.5 \\
\hline Bilateral involvement & $1.7(0.4-6.4)$ & 0.4 \\
\hline Pleural effusion & $5.4(0.9-30.6)$ & 0.1 \\
\hline \multicolumn{3}{|l|}{ Treatment } \\
\hline Chlorambucil & 1 & \\
\hline $\begin{array}{l}\text { Cyclophosphamide based } \\
\text { chemotherapy }\end{array}$ & $0.9(0.1-6.6)$ & 0.9 \\
\hline $\begin{array}{l}\text { Anthracyclin- or fludarabine-based } \\
\text { chemotherapy }\end{array}$ & $2.6(0.4-6)$ & 0.3 \\
\hline Local therapy & $0.2(0.02-2.7)$ & 0.2 \\
\hline Abstention & $4.6(0.4-54)$ & 0.2 \\
\hline
\end{tabular}

Treatment groups are defined in the Methods section. All treatment groups were compared to chlorambucil. ECOG: Eastern Cooperative Oncology Group. \#: by log-rank test, because no death was observed in case of medullar location, the hazard ratio cannot be estimated.

survival in this setting [30], the present series confirms the favourable course of pulmonary MALT lymphoma, with an estimated $72 \% 10$-yr survival $[1,17]$. Since only two of the nine deaths were related to lymphoma, only age and ECOG status were associated with poor outcome. Indeed, none of the lymphoma characteristics were associated with worse survival. PFS, reflecting tumour growth in indolent disease, is probably more useful for the prediction of treatment efficacy than overall survival [15]. The 10-yr PFS was estimated at 36\%, and the factors associated with shorter PFS were mediastinal adenopathy and chemotherapy containing anthracyclin or cyclophosphamide. The mediastinal adenopathy designation in our patients could confer behaviour closer to nodal MZLs rather than MALT lymphoma, in accordance with a previous report [31]. Although there are conflicting results regarding the influence of dissemination on survival [1, 32], an extrapulmonary location was not associated with a shorter PFS or overall survival in this series. Indeed, the impact of extensive evaluation at diagnosis, including bone marrow biopsy and endoscopic examination of the gastrointestinal tract, should be better evaluated in patients who cannot be cured by local therapy.

Two previous large retrospectives studies did not show evidence of different outcomes between patients receiving different treatment modalities, i.e. local therapy, chemotherapy or combined therapy [33, 34]. European Society for Medical Oncology guidelines for gastric non- $H$. pylori MALT lymphoma recommend treatment with radiotherapy for localised lymphoma and chemotherapy using an alkylating agent (chlorambucil or cyclophosphamide) or purine analogue (fludarabine or cladribine) with or without the anti-CD20 monoclonal antibody, rituximab, for disseminated lymphoma [35]. In the present series, patients treated by anthracyclin had worse PFS. However, anthracyclin-based chemotherapy was administered for almost 5 months, while MALT lymphoma with indolent growth may need a maintenance therapy, as recently suggested, with rituximab [36]. This treatment regimen reflects the fact that three patients were treated in 1993 during a period when anthracyclines were recommended [1, 37]. Other patients from the anthracyclin based-chemotherapy group have been treated since 2000. Therapy was chosen by a local physician not always in accordance with newer recommendations. Cyclophosphamide resulted in a lower disease control rate than chlorambucil $(70 \%$ versus $87 \%$, $\mathrm{p}=0.02)$, while both drugs were administered for a similar prolonged duration of almost 15 months. This was corroborated by the lower PFS observed in patients receiving cyclophosphamide-based chemotherapy. For five of the six patients without initial treatment, abstention was chosen because of a limited disease without possible curative radiotherapy. None of these patients presented progression during the median follow-up of 12.5 months, confirming the feasibility of the previously published "watch and wait" policy [38]. Since our study is retrospective and multicentric, conclusions about treatment should be made with caution; however, almost half of the patients (46\%) were treated in the same hospital.

This series confirms the disseminated but indolent nature of pulmonary MALT lymphoma and supports the therapeutic recommendation of chlorambucil chemotherapy for disseminated lymphoma. Prospective studies should be carried out in order to confirm that chlorambucil is the best first-line therapy and to determine its place in the new therapeutic arsenal, which also includes rituximab, fludarabine, cladribine and bortezomib. Further works (i.e. fluorescent in situ hybridisation analysis for API2-MALT1 translocation [39] and universal 16S ribosomal RNA PCR for microbial infection) are in progress that will bring about the better understanding of pulmonary MALT lymphomagenesis, and thus hopefully lead to improvements in the therapeutic strategy.

\section{STATEMENT OF INTEREST}

None declared.

\section{ACKNOWLEDGEMENTS}

Affiliation details are as follows. R. Borie: Service de Pneumologie et Réanimation, Centre de Compétence Maladies Rares Pulmonaires, Hôpital Tenon, Assistance Publique-Hôpitaux de Paris and Faculté de Médecine Pierre et Marie Curie, Université Paris VI, Paris, and Service de Pneumologie A, Centre de Compétence Maladies Rares Pulmonaires, Hôpital Bichat, Assistance Publique-Hôpitaux de Paris, Paris, France; M. Wislez and J. Cadranel: Service de Pneumologie et Réanimation, Centre de Compétence Maladies Rares Pulmonaires, Hôpital Tenon, Assistance Publique-Hôpitaux de Paris and Faculté de Médecine Pierre et Marie Curie, Université Paris VI, Paris, France; G. Thabut and H. Mal: Service de Pneumologie B, Hôpital Bichat, Assistance Publique-Hôpitaux de 
Paris, Paris, France; M. Antoine: Service d'Anatomie-Pathologique, Hôpital Tenon, Assistance Publique-Hôpitaux de Paris, Paris, France; A. Rabbat: Service de Pneumologie et Réanimation, Hôpital Hôtel-Dieu, Assistance Publique-Hopitaux de Paris and Université René Descartes Paris V, Paris, France; L-J. Couderc: Service de Pneumologie, Hôpital Foch, Suresnes and Faculté Paris-Ile de France-Ouest, Versailles, France; I. Monnet: Service de Pneumologie, Centre Hospitalier Intercommunal de Créteil, Créteil, France; H. Nunes: Service de Pneumologie, Centre de Compétence Maladies Rares Pulmonaires, Hôpital Avicenne, Assistance Publique-Hôpitaux de Paris and UPRES EA 2363, Université Paris 13, Bobigny, France; F-X. Blanc: Unité de Pneumologie, Service de Médecine Interne, Hôpital Bicêtre, Assistance Publique-Hôpitaux de Paris, Le Kremlin-Bicêtre, France; A. Bergeron: Service de Pneumologie, Hôpital Saint-Louis, Assistance Publique-Hôpitaux de Paris, Paris, France; D. Dusser: Service de Pneumologie, Hôpital Cochin, Assistance PubliqueHôpitaux de Paris, Paris, France; D. Israël-Biet: Service de Pneumologie, Centre de Compétence Maladies Rares Pulmonaires, Hôpital Européen Georges Pompidou, Assistance Publique-Hôpitaux de Paris, Paris, France; B. Crestani: Service de Pneumologie A, Centre de Compétence Maladies Rares Pulmonaires, Hôpital Bichat, Assistance PubliqueHôpitaux de Paris, Paris, France.

The authors would like to thank J.-P. L'huiller (La Varenne St Hilaire, France) and E. Oksenhendler (Hôpital Saint Louis, Paris, France), who provided outcome data, and T. Chinet (Hôpital Ambroise Paré, Assistance Publique-Hôpitaux de Paris, Boulogne, France), M. Humbert (Hôpital Antoine Béclère, Assistance Publique-Hôpitaux de Paris, Clamart, France), C. Cracco (Hôpital de la Pitié-Salpétrière, Assistance Publique-Hôpitaux de Paris, Paris, France) and C. Chouaid (Hôpital SaintAntoine, Assistance Publique-Hôpitaux de Paris, Paris, France), whose assistance was gratefully received.

\section{REFERENCES}

1 Thieblemont C, Berger F, Dumontet C, et al. Mucosa-associated lymphoid tissue lymphoma is a disseminated disease in one third of 158 patients analyzed. Blood 2000; 95: 802-806.

2 Montalban C, Castrillo JM, Abraira V, et al. Gastric B-cell mucosaassociated lymphoid tissue (MALT) lymphoma. Clinicopathological study and evaluation of the prognostic factors in 143 patients. Ann Oncol 1995; 6: 355-362.

3 Zucca E, Conconi A, Pedrinis E, et al. Nongastric marginal zone Bcell lymphoma of mucosa-associated lymphoid tissue. Blood 2003; 101: 2489-2495.

4 Harris NL, Jaffe ES, Stein H, et al. A revised European-American classification of lymphoid neoplasms: a proposal from the International Lymphoma Study Group. Blood 1994; 84: 1361-1392.

5 Isaacson P, Wright DH. Extranodal malignant lymphoma arising from mucosa-associated lymphoid tissue. Cancer 1984; 53: 25152524.

6 Royer B, Cazals-Hatem D, Sibilia J, et al. Lymphomas in patients with Sjogren's syndrome are marginal zone B-cell neoplasms, arise in diverse extranodal and nodal sites, and are not associated with viruses. Blood 1997; 90: 766-775.

7 Suarez F, Lortholary O, Hermine $\mathrm{O}$, et al. Infection-associated lymphomas derived from marginal zone B cells: a model of antigen-driven lymphoproliferation. Blood 2006; 107: 3034-3044.

8 Wotherspoon AC, Doglioni C, Diss TC, et al. Regression of primary low-grade B-cell gastric lymphoma of mucosa-associated lymphoid tissue type after eradication of Helicobacter pylori. Lancet 1993; 342: 575-577.

9 Thieblemont C, Bastion Y, Berger F, et al. Mucosa-associated lymphoid tissue gastrointestinal and nongastrointestinal lymphoma behavior: analysis of 108 patients. J Clin Oncol 1997; 15: 1624-1630.

10 Roggero E, Zucca E, Pinotti G, et al. Eradication of Helicobacter pylori infection in primary low-grade gastric lymphoma of mucosaassociated lymphoid tissue. Ann Intern Med 1995; 122: 767-769.
11 Montalban C, Manzanal A, Boixeda D, et al. Helicobacter pylori eradication for the treatment of low-grade gastric MALT lymphoma: follow-up together with sequential molecular studies. Ann Oncol 1997; 8: Suppl. 2, 37-39.

12 Savio A, Franzin G, Wotherspoon AC, et al. Diagnosis and posttreatment follow-up of Helicobacter pylori-positive gastric lymphoma of mucosa-associated lymphoid tissue: histology, polymerase chain reaction, or both? Blood 1996; 87: 1255-1260.

13 Isaacson PG, Müller-Hermelink HK, Piris MA, et al. Extranodal marginal zone B-cell lymphoma of maucosa-associated lymphoid tissue (MALT lymphoma). In: Press I, Jaffe ES, Harris NL, et al., eds. Pathology and Genetics: Tumours of Haematopoietic Tissues. World Health Organization Classification of Tumours. Lyon, WHO, 2001; pp. 157-160.

14 Wislez M, Cadranel J, Antoine M, et al. Lymphoma of pulmonary mucosa-associated lymphoid tissue: $\mathrm{CT}$ scan findings and pathological correlations. Eur Respir J 1999; 14: 423-429.

15 Cheson BD, Pfistner B, Juweid ME, et al. Revised response criteria for malignant lymphoma. J Clin Oncol 2007; 25: 579-586.

16 Kinder BW, Collard HR, Koth L, et al. Idiopathic nonspecific interstitial pneumonia: lung manifestation of undifferentiated connective tissue disease? Am J Respir Crit Care Med 2007; 176: 691-697.

17 Cordier JF, Chailleux E, Lauque D, et al. Primary pulmonary lymphomas. A clinical study of 70 cases in nonimmunocompromised patients. Chest 1993; 103: 201-208.

18 Bae YA, Lee KS, Han J, et al. Marginal zone B-cell lymphoma of bronchus-associated lymphoid tissue: imaging findings in 21 patients. Chest 2008; 133: 433-440.

19 Campo E, Chott A, Kinney MC, et al. Update on extranodal lymphomas. Conclusions of the Workshop held by the EAHP and the SH in Thessaloniki, Greece. Histopathology 2006; 48: 481-504.

20 de Boer JP, Hiddink RF, Raderer M, et al. Dissemination patterns in non-gastric MALT lymphoma. Haematologica 2008; 93: 201-206.

21 Hoffmann $\mathrm{M}$, Wohrer S, Becherer A, et al. ${ }^{18} \mathrm{~F}-$ Fluoro-deoxyglucose positron emission tomography in lymphoma of mucosaassociated lymphoid tissue: histology makes the difference. Ann Oncol 2006; 17: 1761-1765.

22 Radan L, Fischer D, Bar-Shalom R, et al. FDG avidity and PET/CT patterns in primary gastric lymphoma. Eur J Nucl Med Mol Imaging 2008; 35: 1424-1430.

23 Kassan SS, Thomas TL, Moutsopoulos HM, et al. Increased risk of lymphoma in sicca syndrome. Ann Intern Med 1978; 89: 888-892.

24 Ekstrom Smedby K, Vajdic CM, Falster M, et al. Autoimmune disorders and risk of non-Hodgkin lymphoma subtypes: a pooled analysis within the InterLymph Consortium. Blood 2008; 111: 40294038.

25 Nakatsuka S, Yao M, Hoshida Y, et al. Pyothorax-associated lymphoma: a review of 106 cases. J Clin Oncol 2002; 20: 4255-4260.

26 Katzenstein AL, Peiper SC. Detection of Epstein-Barr virus genomes in lymphomatoid granulomatosis: analysis of 29 cases by the polymerase chain reaction technique. Mod Pathol 1990; 3: 435-441.

27 Boulanger E, Gerard L, Gabarre J, et al. Prognostic factors and outcome of human herpesvirus 8-associated primary effusion lymphoma in patients with AIDS. J Clin Oncol 2005; 23: 4372-4380.

28 Hansen JM, Wildner-Christensen M, Hallas J, et al. Effect of a community screening for Helicobacter pylori: a 5-Yr follow-up study. Am J Gastroenterol 2008; 103: 1106-1113.

29 Chan WY, Chan EK, Chow JH. Epstein-Barr virus-associated gastric lymphomas are distinct from mucosa-associated lymphoid tissue-type lymphomas: genetic abnormalities of p53 gene. Diagn Mol Pathol 2001; 10: 153-160.

30 Kalpadakis C, Pangalis GA, Vassilakopoulos TP, et al. Non-gastric extra-nodal marginal zone lymphomas - a single centre experience on 76 patients. Leuk Lymphoma 2008; 49: 2308-2315.

31 Thieblemont C. Non-MALT marginal zone lymphomas. Ann Oncol 2008; 19: Suppl. 4, iv70-iv73. 
32 Arcaini L, Burcheri S, Rossi A, et al. Nongastric marginal-zone Bcell MALT lymphoma: prognostic value of disease dissemination. Oncologist 2006; 11: 285-291.

33 Pinotti G, Zucca E, Roggero E, et al. Clinical features, treatment and outcome in a series of 93 patients with low-grade gastric MALT lymphoma. Leuk Lymphoma 1997; 26: 527-537.

34 Thieblemont C, Dumontet C, Bouafia F, et al. Outcome in relation to treatment modalities in 48 patients with localized gastric MALT lymphoma: a retrospective study of patients treated during 19762001. Leuk Lymphoma 2003; 44: 257-262.

35 Zucca E, Dreyling M. Gastric marginal zone lymphoma of MALT type: ESMO clinical recommendations for diagnosis, treatment and follow-up. Ann Oncol 2008; 19: Suppl. 2, ii70-ii71.
36 Hainsworth JD, Litchy S, Burris HA 3rd, et al. Rituximab as firstline and maintenance therapy for patients with indolent nonHodgkin's lymphoma. J Clin Oncol 2002; 20: 4261-4267.

37 Coiffier B, Thieblemont C, Felman P, et al. Indolent nonfollicular lymphomas: characteristics, treatment, and outcome. Semin Hematol 1999; 36: 198-208.

38 Troch M, Streubel B, Petkov V, et al. Does MALT lymphoma of the lung require immediate treatment? An analysis of 11 untreated cases with long-term follow-up. Anticancer Res 2007; 27: 3633-3637.

39 Remstein ED, James CD, Kurtin PJ. Incidence and subtype specificity of API2-MALT1 fusion translocations in extranodal, nodal, and splenic marginal zone lymphomas. Am J Pathol 2000; 156: $1183-1188$. 